\title{
Panoscopic approach for high-performance Te-doped skutterudite
}

\author{
Tao Liang ${ }^{1}$, Xianli Su${ }^{1}$, Yonggao Yan ${ }^{1}$, Gang Zheng ${ }^{1}$, Xiaoyu She ${ }^{1}$, Yonghui You ${ }^{1}$, Ctirad Uher ${ }^{2}$, \\ Mercouri G Kanatzidis ${ }^{3}$ and Xinfeng Tang ${ }^{1}$
}

One-step plasma-activated sintering (OS-PAS) fabrication of single-phase high-performance $\mathrm{CoSb}_{3}$-based skutterudite thermoelectric material with a hierarchical structure on a time scale of a few minutes is first reported here. The formation mechanism of the $\mathrm{CoSb}_{3}$ phase and the effects of the current and pressure fields on the phase transformation and microstructure evolution are studied in the one-step PAS process. The application of the panoscopic approach to this system and its effect on the transport properties are investigated. The results show that the hierarchical structure forms during the formation of the skutterudite phase under the effects of both current and sintering pressure. The samples fabricated by the OS-PAS technique have defined hierarchical structures, which scatter phonons more intensely over a broader range of frequencies and significantly reduce the lattice thermal conductivity. High-performance bulk Te-doped skutterudite with the maximum $Z T$ of 1.1 at $820 \mathrm{~K}$ for the composition $\mathrm{CoSb}_{2.875} \mathrm{Te}_{0.125}$ was obtained. Such high $Z T$ values rival those obtained from single filled skutterudites. This newly developed OS-PAS technique enhances the thermoelectric performance, dramatically shortens the synthesis period and provides a facile method for obtaining hierarchical thermoelectric materials on a large scale. NPG Asia Materials (2017) 9, e352; doi:10.1038/am.2017.1; published online 24 February 2017

\section{INTRODUCTION}

Thermoelectric technology uses solid-state semiconductors, which can directly convert heat into electricity and vice versa using the Seebeck effect for power generation and Peltier effect for cooling. The efficiency of thermoelectric materials is governed by the dimensionless figure of merit $Z T=\alpha^{2} \sigma T / \kappa$, where $\alpha, \sigma, T$ and $\kappa$ are the Seebeck coefficient, electrical conductivity, absolute temperature and thermal conductivity, respectively. Many studies have been conducted in the past decades ${ }^{1-5}$ to enhance the thermoelectric properties with a focus on improving the power factor and decreasing the thermal conductivity. Because of the high electrical transport performance ${ }^{6,7}$ and relatively good mechanical properties, ${ }^{8}$ skutterudites are considered notably promising for commercial power generation thermoelectric applications $s^{9,10}$ in the temperature range of 500-900 K..$^{6,7,11,12}$ However, the disadvantage of skutterudites is their notably high lattice thermal conductivity of $>10 \mathrm{~W} \mathrm{~m}^{-1} \mathrm{~K}^{-1}$. To obtain a higher conversion efficiency, the thermal conductivity must be further reduced.

The thermal conductivity of skutterudites derives from the contributions of phonons with a notably broad range of frequencies and mean free paths (MFPs). ${ }^{13-17}$ Those phonons are primarily scattered by features in the structure (dopant, nanostructures and so on) that are comparable in size to the MFP of the phonons. For example, the high-frequency (short-wavelength) phonons tend to be scattered more effectively by atomic-scale point defects, that is, doping foreign species at the sites of $\mathrm{Co}^{18,19}$ and $\mathrm{Sb}^{7,11,20,21}$ or introducing filler atoms ${ }^{22-24}$ into the structural voids (cages) of $\mathrm{CoSb}_{3}$. The medium-frequency phonons with medium MFP of several to hundreds of nanometers are significantly scattered by nanoprecipitates or other nanoscale heterogeneities. Thus nanostructures introduced by the melting-spinning ${ }^{23,24}$ (MS) technique or ball milling ${ }^{20}$ can dramatically reduce the thermal conductivity of skutterudites. However, the low-frequency phonons with long MFPs generally remain unaffected.

Additional and remarkable decreases in lattice thermal conductivity $\left(\kappa_{\mathrm{L}}\right)$ may be obtained if the phonons with longer MFPs can be scattered by features such as mesoscale grains and grain boundaries (with sizes of hundreds of nanometers to micrometers). Therefore, structures that contain heterogeneities on multiple length scales (atomic scale, nanoscale and mesoscale) are highly desired. This demand represents a panoscopic approach ${ }^{25,26}$ to substantially decrease $\kappa_{\mathrm{L}}$ and improve $Z T$ in skutterudite bulk materials. Experimentally, the panoscopic structure $e^{25,26}$ has been realized by Kanatzidis and co-workers to enhance the thermoelectric performance of $\mathrm{PbTe}$ because of phonon scattering over a broad frequency, which suppresses the thermal conductivity. ${ }^{1}$ However, for the class of skutterudites, notably little attention has been focused on panoscopic structures to improve the thermoelectric properties.

${ }^{1}$ State Key Laboratory of Advanced Technology for Materials Synthesis and Processing, Wuhan University of Technology, Hubei, China; ${ }^{2}$ Department of Physics, University of Michigan, Ann Arbor, MI, USA and ${ }^{3}$ Department of Chemistry, Northwestern University, Evanston, IL, USA

Correspondence: Professor X Su or X Tang, State Key Laboratory of Advanced Technology for Materials Synthesis and Processing, Wuhan University of Technology, 122 Luoshi Road, Wuhan, Hubei 430070, China.

E-mail: suxianli@whut.edu.cn or tangxf@whut.edu.cn

Received 7 June 2016; revised 10 November 2016; accepted 15 November 2016 
Plasma-activated sintering (PAS) is a particular hot-pressing process that induces two distinct effects: (i) heat is internally generated based on Joule heating, which consolidates the material to its near theoretical density; and (ii) the added heat energy may assist in a phase transformation to the desired phase. In the former case, traditionally, the morphology of the structure is unchanged and only its density increases. It cannot produce the desired hierarchical structure. In the latter case, the application of a direct current can actually modulate the sample morphology because the applied current may increase the rate of nucleation and refine the grains by decreasing the thermodynamic barrier during the formation of a particular phase. ${ }^{27-29}$ In addition, when an electrical current is applied during the sintering process, the temperature profile is often inhomogeneous because of the nonuniform distribution of the current that passes through the powdered compact. This inhomogeneity results in uneven nucleation, and some grains experience an abnormal growth rate; therefore, the resulting structure has a wider range of grain sizes.

In this paper, we describe the one-step PAS (OS-PAS) technique, which is applied to synthesize Te-doped $\mathrm{CoSb}_{3}$-based skutterudites. We demonstrate that the thermoelectric performance of the material is improved by introducing a hierarchical structure induced by the PAS process (herein, PAS can also be referred to as spark plasma sintering (SPS) because PAS is notably similar to SPS). We show that the direct current and applied pressure exert considerable effects on the phase transformations and microstructure evolution. Controllable formation of hierarchical structures is realized, and their effect on the thermoelectric properties is documented. To reveal the effects of the panoscopic approach (forming a hierarchical structure) on the reduction of $\kappa_{\mathrm{L}}$ of skutterudite, we compare the essential features of Te-doped skutterudite bulk materials with different microstructure configurations synthesized by melting quenching-annealing-plasma activated sintering (MQ-AN-PAS), MS-PAS and the newly developed OS-PAS. These three synthesis techniques for skutterudite are displayed in Supplementary Figure S1.

\section{METHODS}

Pure elemental Co (>99.9\% 200 mesh powder), Sb (99.9999\% 200 mesh powder) and $\mathrm{Te}(99.9999 \% 200$ mesh powder) were weighed according to the nominal composition of $\mathrm{CoSb}_{3-x} \mathrm{Te}_{x}(x=0,0.075,0.1,0.125,0.15)$. The elemental powders were mixed and ground by hand milling in an agate mortar. The obtained powder mixture was loaded into a graphite die with a diameter of
$15 \mathrm{~mm}$ and protected with graphitic carbon paper. This assembly was placed in the PAS device (PAS-III-Ed, Elenix, Japan). The sintering current, heating rate, sintering temperature, sintering pressure and holding time were 500-550 A, $100{ }^{\circ} \mathrm{C} \min ^{-1}, 550{ }^{\circ} \mathrm{C}, 40 \mathrm{MPa}$ and $5 \mathrm{~min}$, respectively. To eliminate the effect of the grain size distribution in the starting powders of $\mathrm{Co}$ and $\mathrm{Sb}$, all precursor powders were sieved through a 200 mesh.

The resulting bulk structures were examined by powder X-ray diffractometry (XRD; PANalytical (Almelo, The Netherlands): X'Pert PRO, Cu Ko). The morphology and elemental distributions were determined by back-scattered electron imaging (field-emission scanning electron microscope (FESEM), Hitachi, Tokyo, Japan, SU8020) and energy dispersive X-ray (EDX) analysis (Bruker, Billerica, MA, USA), respectively. The microstructure of the obtained samples was studied with transmission electron microscopy (TEM) using a JEM-2100F instrument, JEOL (Tokyo, Japan) and FESEM. The composition of nano-inclusions and the surrounding matrix were qualitatively determined by energy dispersive X-ray spectroscopy using an EDAX AMETEK (Berwyn, PA, USA). To prepare specimens for TEM, the bulk material was ion-milled using a Gatan Model 691 apparatus (Pleasanton, CA, USA). The electrical conductivity and Seebeck coefficient were simultaneously measured using a standard fourprobe method by ZEM-3, ULVAC-RIKO (Kanagawa, Japan), in a He atmosphere. The carrier concentration of the specimens was obtained from the Hall coefficient, which was measured using the Physical Property Measuring System. The thermal conductivity was calculated from the measured thermal diffusivity $D$, specific heat $C_{\mathrm{p}}$ and density $d$ according to the relationship $\kappa=D C_{\mathrm{p}} d$. The thermal diffusivity and specific heat were determined using a laser flash method NETZSCH: LFA 457 (Selb, Germany) and a power-compensating differential scanning calorimeter TA: DSC Q20 (Delaware, PA, USA), respectively, in an Ar atmosphere. The density of the samples was measured by using Archimedes principle as shown in Supplementary Table S1. All measurements were performed in the temperature range of $300-820 \mathrm{~K}$.

\section{RESULTS}

Powder XRD patterns of the $\mathrm{CoSb}_{3-x} \mathrm{Te}_{x}(x=0-0.15)$ specimens after the OS-PAS process at a sintering temperature of $550^{\circ} \mathrm{C}$ for $5 \mathrm{~min}$ are shown in Figure 1. The XRD patterns in the range of $10-80^{\circ}$ are displayed in Figure 1a and demonstrate that all samples after PAS at this sintering temperature are single-phase structures, which match the standard JC-PDF card of $\mathrm{CoSb}_{3}$ (JCPDS\# 01-083-0055). As shown in Figure $1 \mathrm{~b}$, all diffraction peaks gradually shift towards lower angles when the content of Te increases, which indicates that the lattice parameter increases when Te substitutes for $\mathrm{Sb}$. The data indicate that the OS-PAS process at a sintering temperature of at least $550{ }^{\circ} \mathrm{C}$ yields the desired $\mathrm{CoSb}_{3}$ phase in $<12 \mathrm{~min}$.
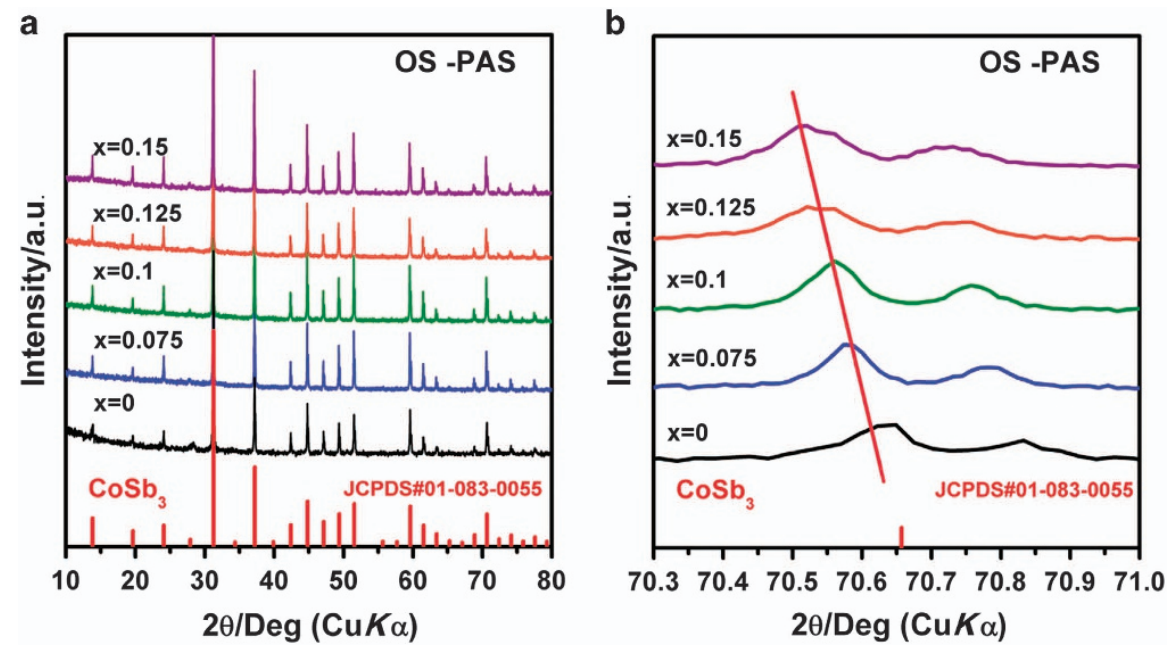

Figure 1 Powder X-ray diffraction patterns of $\mathrm{CoSb}_{3-x} \mathrm{Te}_{x}(x=0-0.15)$ specimens after the OS-PAS process. (a) $\mathrm{XRD}$ patterns in the range $10^{\circ}-80^{\circ}$, (b) peak shifts at high angles due to doping by Te. 
a

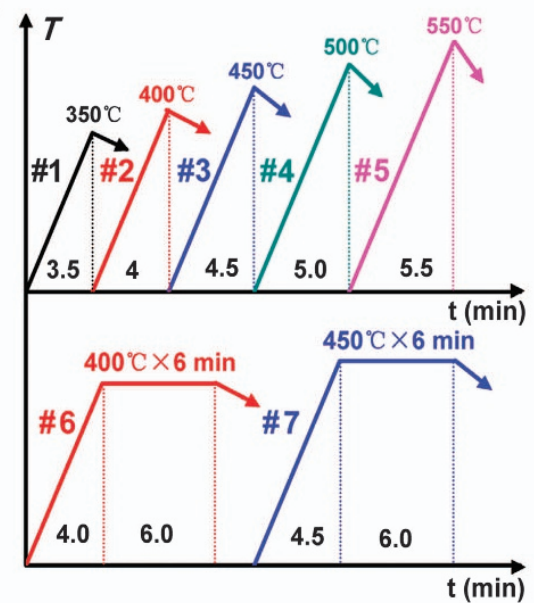

b

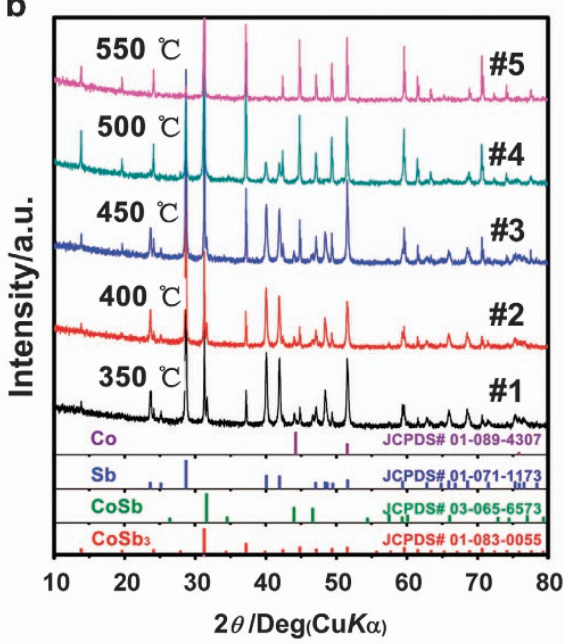

d

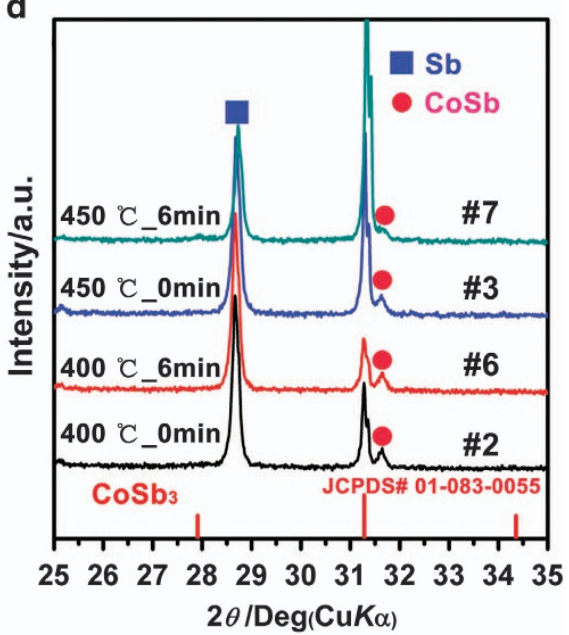

Figure 2 XRD patterns of samples synthesized by OS-PAS at different stages of the applied PAS process. (a) The PAS processing parameters for samples \#1-\#7. The sintering pressure for all samples is $40 \mathrm{MPa}$; (b) XRD patterns of bulk samples described in a; (c) an expanded angular scale of $\mathbf{b}$ focusing on the peaks of $\mathrm{Sb}$ and $\mathrm{CoSb}$; (d) an expanded angular scale of XRD patterns for samples heated to $400{ }^{\circ} \mathrm{C}$, respectively, $450{ }^{\circ} \mathrm{C}$ and either immediately cooled down (samples \#2 and \#3) or held there for 6 min (samples \#6 and \#7).

To gain insight into the formation process of the $\mathrm{CoSb}_{3}$ phase and follow the overall reaction $\mathrm{Co}+3 \mathrm{Sb} \rightarrow \mathrm{CoSb}_{3}$, we collected $\mathrm{XRD}$ patterns of synthesized materials by OS-PAS at different sintering temperatures (Figure 2). The PAS processing parameters, that is, the temperature and time during which the pressure of $40 \mathrm{MPa}$ was applied to each sample, which were labeled \#1-\#7, are shown in Figure 2a. Samples \#1-\#5 were heated to their target temperatures of $350-550{ }^{\circ} \mathrm{C}$ with the heating rate of $100 \mathrm{~K} \mathrm{~min}^{-1}$ and immediately cooled to room temperature. Samples \#6 and \#7 were also heated to their respective target temperatures but maintained there for $6 \mathrm{~min}$ before being cooled to room temperature. Figure $2 \mathrm{~b}$ shows the XRD patterns obtained on materials that were subjected to the PAS stages described in Figure 2a. The results show that the intensity of the $\mathrm{Sb}$ diffraction peaks decreases, whereas the intensity of the $\mathrm{CoSb}_{3}$ peaks steadily increases with increasing sintering temperature. This result suggests that the reaction is gradually completed with the increase in sintering temperature. The elemental $\mathrm{Sb}$ gradually reacts with $\mathrm{Co}$; when the temperature reaches $550^{\circ} \mathrm{C}$, essentially all $\mathrm{Sb}$ and Co have reacted to form $\mathrm{CoSb}_{3}$ skutterudite. Therefore, we surmise that the required critical temperature to complete the reaction of $\mathrm{Sb}$ with
Co and form $\mathrm{CoSb}_{3}$ is approximately $550^{\circ} \mathrm{C}$. Consequently, all samples that were prepared for transport measurements were sintered at $550{ }^{\circ} \mathrm{C}$.

To look more closely at the reaction between $\mathrm{Co}$ and $\mathrm{Sb}$, we show the expanded XRD patterns of Figure $2 b$ in Figure $2 c$. The data show that (a) in the initial reaction stage $\left(T<350^{\circ} \mathrm{C}\right)$, Co and $\mathrm{Sb}$ begin to react to form $\mathrm{CoSb}_{3}$ and a small amount of $\mathrm{CoSb}$; however, the main phase remains pure $\mathrm{Sb}$; (b) when the sintering temperature increases $\left(350{ }^{\circ} \mathrm{C}<T<500^{\circ} \mathrm{C}\right)$, the intensity of the CoSb peak gradually diminishes until it completely disappears; the most probable scenario for this occurrence is the reaction of $\mathrm{CoSb}$ with $\mathrm{Sb}$ : $\mathrm{CoSb}+2 \mathrm{Sb} \rightarrow \mathrm{CoSb}_{3}$; (c) above $500{ }^{\circ} \mathrm{C}$, the amount of $\mathrm{CoSb}_{3}$ phase increases until $\mathrm{Sb}$ and $\mathrm{Co}$ are fully reacted and only the $\mathrm{CoSb}_{3}$ phase remains. Interestingly, we detect no trace of $\mathrm{CoSb}_{2}$ phase in the entire OS-PAS synthesis, which indicates that the reaction $\mathrm{Co}+2 \mathrm{Sb} \rightarrow \mathrm{CoSb}_{2}$ is inhibited; thus one does not encounter the time-consuming peritectic transformation of $\mathrm{CoSb}_{2}$ to $\mathrm{CoSb}_{3}$. Applying the OS-PAS technique to prepare $\mathrm{CoSb}_{3}$ shortens the synthesis period and removes the troublesome metallic $\mathrm{CoSb}_{2}$ impurity phase, which may cause a serious deterioration of the thermoelectric performance if present. 

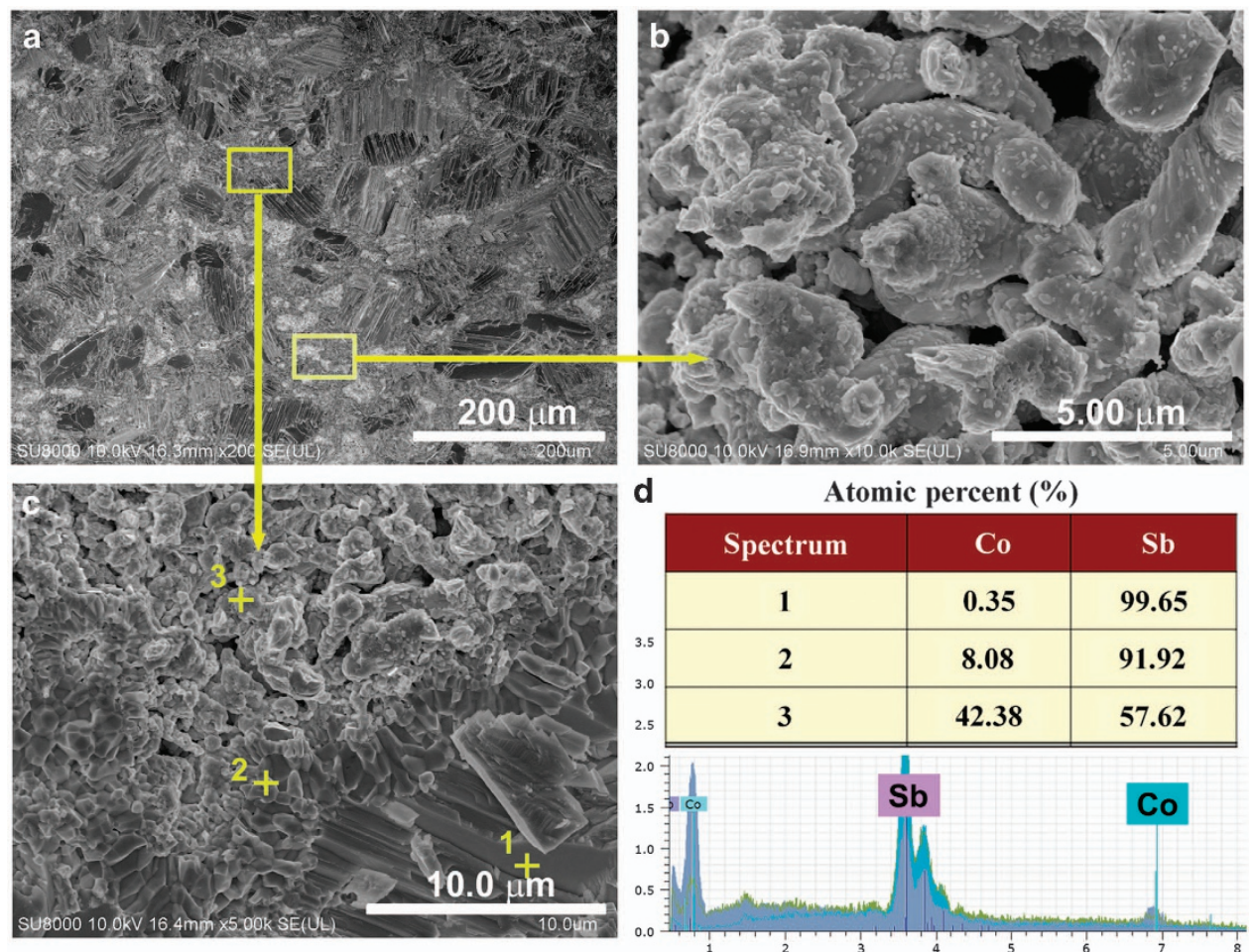

Figure $3 \mathrm{SEM}$ images of fractured surfaces of $\mathrm{CoSb}_{3}$ produced by OS-PAS processing under pressure of $40 \mathrm{MPa}$. Samples were heated to $450{ }^{\circ} \mathrm{C}$ with a heating rate of $100 \mathrm{~K} \mathrm{~min}^{-1}$ and immediately cooled down to room temperature. (a) Low magnification image; (b) an enlarged area from the marked yellow rectangle in a; (c) an enlarged area showing coarse grains from other yellow rectangle in a; (d) EDX analysis from the different locations labeled as 1 , 2 and 3 in $\mathbf{c}$.

However, the exact reason of why and how the OS-PAS process inhibits the $\mathrm{CoSb}_{2}$ phase formation is unclear and may require further studies.

The experiments in Figures $2 \mathrm{a}$ and $\mathrm{b}$ identify two important reactions

$$
\mathrm{Co}+\mathrm{Sb} \rightarrow \mathrm{CoSb}
$$

and

$$
\mathrm{CoSb}+2 \mathrm{Sb} \rightarrow \mathrm{CoSb}_{3}
$$

To further study the CoSb formation (equation (1)) and its conversion to the skutterudite phase (equation (2)), samples \#6 and \#7 were heated to the target temperature of 400 and $450^{\circ} \mathrm{C}$, respectively, and maintained there for $6 \mathrm{~min}$ before being cooled to room temperature. This process is sketched at the bottom of Figure $2 \mathrm{a}$, and the corresponding $\mathrm{XRD}$ patterns of the product are presented in Figure 2d. Clearly, the CoSb phase is present when the sintering temperature in the PAS process is $400^{\circ} \mathrm{C}$, regardless of any waiting time at this temperature (see the XRD pattern of sample \#2). Interestingly, the characteristic peak of CoSb slightly increases relative to the $\mathrm{CoSb}_{3}$ peak when the sample is parked for $6 \mathrm{~min}$ at $400{ }^{\circ} \mathrm{C}$ (the pattern of sample \#6). This result suggests that the reaction rate of equation (2) at this temperature is lower than that of equation (1), which continuously increases the CoSb amount. When the sintering temperature reaches $450{ }^{\circ} \mathrm{C}$ (sample \#3), the product with no holding time at this temperature still contains the CoSb phase. However, the intensity of the characteristic diffraction peak of CoSb significantly decreases after the sample is held at this temperature for $6 \mathrm{~min}$ (sample \#7). This result indicates that CoSb begins to convert to $\mathrm{CoSb}_{3}$ and that the reaction in equation (2) becomes the overall ratedetermining event. In summary, the key factor that initiates the formation and conversion of the CoSb phase is the sintering temperature with the holding time for the diffusion process (supply of $\mathrm{Sb}$ ) to occur.

The identical samples as above were used to characterize the morphological evolution of $\mathrm{CoSb}_{3}$, which was probed using SEM. Because of the incomplete uniformity and density of the stoichiometric mixture of the raw $\mathrm{Co}$ and $\mathrm{Sb}$ powders, the electric current applied during the PAS process cannot fully uniformly flow though the compacted powder. ${ }^{30}$ Thus there are localized regions of higher Joule heating, where the temperature is enhanced beyond the melting point of $\mathrm{Sb}$, although the average measured temperature on the graphite die is well below the melting point of Sb. The resulting local melting of $\mathrm{Sb}$ and its subsequent crystallization when the ingot is cooled to room temperature leave its specific imprint on the sample morphology and initiate a reaction sequence, which eventually forms the desired single-phase $\mathrm{CoSb}_{3}$ material.

We illustrate the key features of this process using SEM images. Figure 3 displays the SEM images of the fractured surfaces of sample \#3, which was sintered at $450^{\circ} \mathrm{C}$ with no holding time (sample described in Figure 2). As shown in Figure 3a, many coarse grains with the size of $70-150 \mu \mathrm{m}$ are distributed in the bulk sample with welldefined layer planes. EDX shows that those grains are essentially pure $\mathrm{Sb}$. Interestingly, these $\mathrm{Sb}$ grains are much larger than the initial $\mathrm{Sb}$ grains compared with the results of Supplementary Figure S2a. Hence, partial $\mathrm{Sb}$ was in the liquid state during the PAS process, which is possible because of the nonuniformity in the current passing through the compressed powder, which locally heats some regions beyond the melting point of Sb. When Sb melts, it reacts with the neighboring Co atoms and forms both $\mathrm{CoSb}$ and $\mathrm{CoSb}_{3}$ phases. The actual fraction of each phase depends on the sintering conditions. 

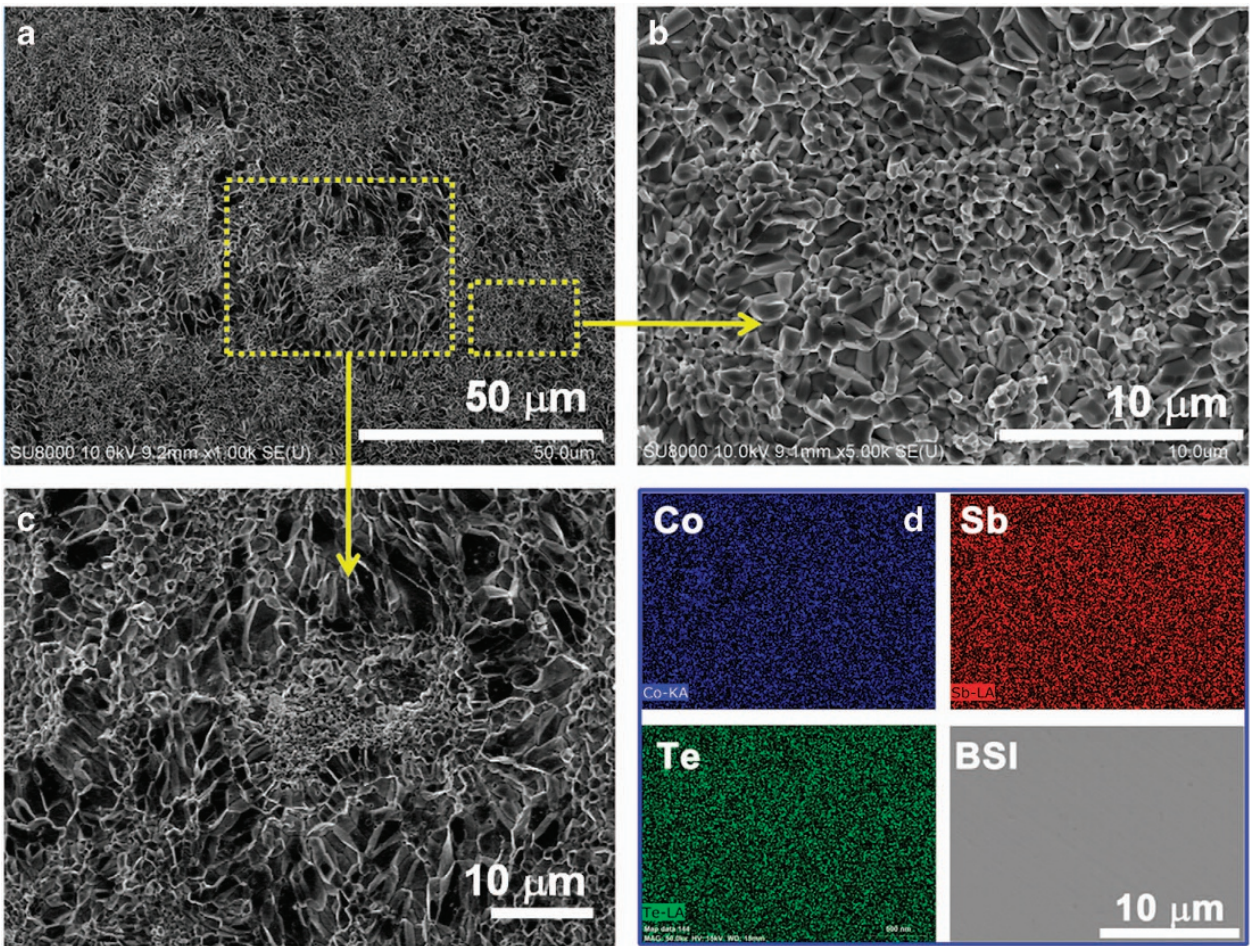

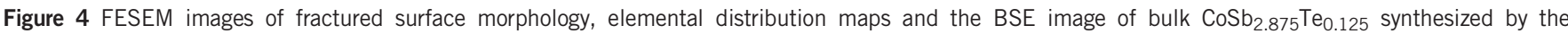
OS-PAS process. (a) Low magnification image; (b) an enlarged area of the outside region marked by the yellow rectangle in a; (c) an enlarged area of the inside region delineated by another yellow rectangle in $\mathbf{a}$; (d) EDX elemental distribution maps and the BSE image of $\mathrm{CoSb}_{2.875} \mathrm{Te}_{0.125} \mathrm{prepared}$ by the OS-PAS process.

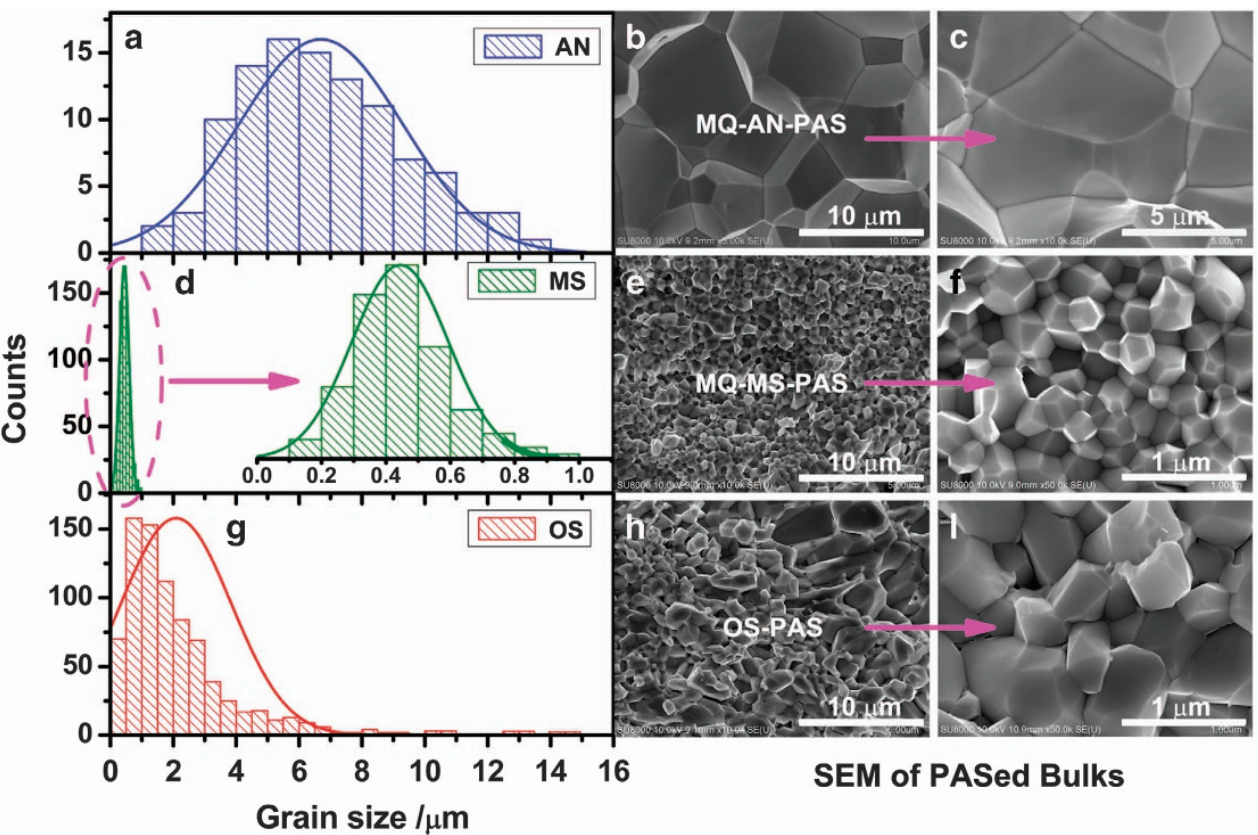

Figure 5 Grain size distributions calculated by the linear intercept method and the corresponding FESEM images. (a-c) Results for CoSb $b_{3}$ prepared by MQ-AN-PAS; (d-f) results for $\mathrm{CoSb}_{3}$ prepared by MS-PAS; (g-I) results for $\mathrm{CoSb}_{3}$ prepared by OS-PAS.

At low temperatures such as $400{ }^{\circ} \mathrm{C}$, the $\mathrm{CoSb}$ phase is preferred as evidenced in the XRD data in Figure 2c, where the skutterudite peak is anomalously small and the peak of unreacted $\mathrm{Sb}$ is high. However, when the sintering temperature increases, the rate of formation of
$\mathrm{CoSb}_{3}$ significantly exceeds that of CoSb and the skutterudite phase grows at the expense of the CoSb phase. The passage of the current appears to assist this process and likely also contributes to a high rate of nucleation of the skutterudite phase. ${ }^{27-29}$ 

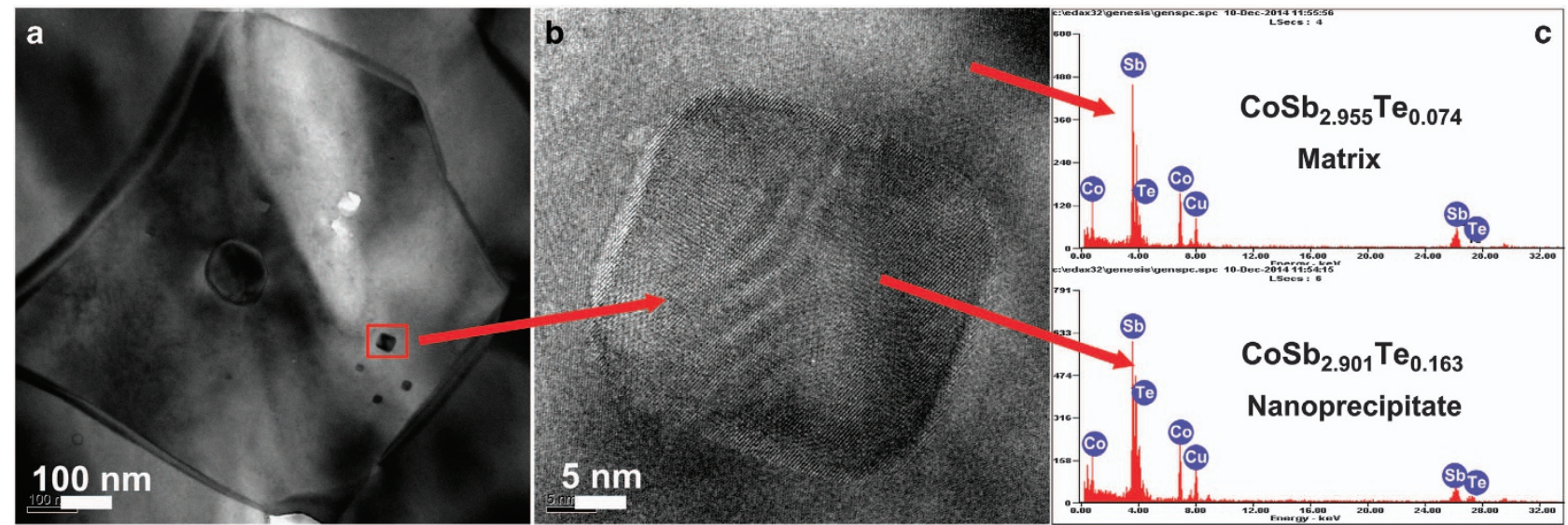

Figure 6 HRTEM and EDX of bulk $\mathrm{CoSb}_{2.875} \mathrm{Te}_{0.125}$ synthesized by the OS-PAS technique. (a) Low-magnification TEM image; (b) high-resolution TEM image; (c) EDX analysis of the matrix and nanoprecipitates, respectively.

A representative region of Figure $3 \mathrm{a}$ is further enlarged and shown in Figure 3c. Meanwhile, the EDX results with respect to the position of Nos.1-3 in Figure 3c are provided in Figure 3d. The area around No. 1 with typical layer planes is identified as pure $\mathrm{Sb}$, and the region marked by No. 2 with much finer grains is mostly Sb that is about to participate in the reaction of $\mathrm{Co}$ and $\mathrm{Sb}$. $\mathrm{CoSb}_{3}$ may also form in region No. 2 because of the fact that Co is surrounded by an extremely rich $\mathrm{Sb}$ environment. The region labeled as No. 3 has the morphology in Figure $3 \mathrm{~b}$ and features numerous unconnected grains, which are a Co-rich phase and presumably a mixture of $\mathrm{Co}, \mathrm{CoSb}$ and $\mathrm{CoSb}_{3}$ according to XRD and EDX. This phase assignment makes sense because, according to the phase diagram, ${ }^{31}$ there are only three stable phases in the Co-Sb system: metallic CoSb, metallic $\mathrm{CoSb}_{2}$, and semiconducting $\mathrm{CoSb}_{3}$. Because the $\mathrm{CoSb}_{2}$ phase appears to be inhibited and absent from all of the XRD scans regardless of the sintering temperature, the Co-rich phase should be a mixture of unreacted $\mathrm{Co}, \mathrm{CoSb}$ and $\mathrm{CoSb}_{3}$. Examples are provided by the SEM images of both morphology and composition evolution at different sintering temperatures and are presented in Supplementary Figures S2-S4.

Figure 4 displays the sample morphology obtained using FESEM and elemental distributions and the back scattered electrons (BSE) image of bulk $\mathrm{CoSb}_{2.875} \mathrm{Te}_{0.125}$ compounds, which were synthesized using the OS-PAS technique at a sintering temperature of $550^{\circ} \mathrm{C}$ and an applied current. Figure 4 a shows several annular regions with the diameter of approximately $50 \mu \mathrm{m}$, which contain grains as small as $100 \mathrm{~nm}$ and are surrounded by coarser grains with sizes of $5-15 \mu \mathrm{m}$, which indicates a multiscale grain structure of skutterudite. FESEM images of these contrasting regions are shown with a higher magnification in Figures $4 \mathrm{~b}$ and c. The results show that, regardless of the grain size, all grains tightly pack with good crystallinity, and the OS-PAS process yields highly dense bulk samples (all relativity densities are $>99 \%$ ). The EDX elemental distributions and BSE image of bulk $\mathrm{CoSb}_{3-} \mathrm{Te}_{x}$ prepared by the OS-PAS synthesis at $550{ }^{\circ} \mathrm{C}$ under current are displayed in Figure $4 \mathrm{~d}$. The elemental distribution results show that $\mathrm{Co}, \mathrm{Sb}$ and $\mathrm{Te}$ in bulk $\mathrm{CoSb}_{2.85} \mathrm{Te}_{0.15}$ compounds are homogeneously distributed, and the BSE contrast is consistent with no traces of any impurity phase, which demonstrates that $\mathrm{Te}$ has entered the $\mathrm{CoSb}_{3}$ crystal lattice and that the OS-PAS process indeed yields a highly homogeneous single-phase skutterudite.

Figure 5 presents the grain size distribution, which was calculated using the linear intercept method and the corresponding FESEM images. Figures $5 \mathrm{a}-\mathrm{c}$ show results for the $\mathrm{CoSb}_{3}$ sample prepared by MQ-AN-PAS. The grain size is notably coarse and extends over the range of $\sim 2-15 \mu \mathrm{m}$. Thus this traditional method of processing yields larger grains; therefore, such samples have a higher lattice thermal conductivity. As shown in Figures $5 \mathrm{~d}-\mathrm{f}$, the grain size of $\mathrm{CoSb}_{3}$ prepared by MS-PAS is notably fine and reaches a submicron range of $100-800 \mathrm{~nm}$. Such relatively fine grain structure is expected to effectively scatter phonons of similar sized MFPs and reduces the lattice thermal conductivity. The results for $\mathrm{CoSb}_{3}$ fabricated by OS-PAS are displayed in Figures $5 \mathrm{~g}-1$. Here the grain size distribution is broader, namely, $100 \mathrm{~nm}-15 \mu \mathrm{m}$, which demonstrates that the OS-PAS technique can result in a more hierarchically structured $\mathrm{CoSb}_{3}$. Such multiscale structures scatter phonons over an even broader range of frequencies and effectively decrease the lattice thermal conductivity.

To obtain further structural information on the nanoscale from samples prepared by the OS-PAS process, we characterized bulk $\mathrm{CoSb}_{2.875} \mathrm{Te}_{0.125}$ by TEM. Figure 6a displays the TEM image at low magnification, and Figure $6 \mathrm{~b}$ is its high-resolution image. Nanoparticles of 5-100 nm are evident. Figure $6 \mathrm{~b}$ highlights a square-shaped nanoparticle with clearly defined boundaries. Compared with the matrix, the EDX analysis (see Figure 6c) identifies the nanoparticle as relatively rich in Te and notably slightly deficient in Sb. Precipitation of Te-rich nanoparticles is possible because of a rapid and non-equilibrium ${ }^{32}$ nature of the OS-PAS process. Combined with the aforementioned range of grain sizes and the point defects introduced by $\mathrm{Te}$ substitution, samples fabricated by OS-PAS clearly represent hierarchically structured materials with structural imperfections that cover all relevant length scales (that is, atomic scale, nanoscale and mesoscale). These structures are expected to impede the flow of phonons over a notably broad range of frequencies and result in exceptionally low lattice thermal conductivities. $1,26,33,34$

In this section, we address the effect of different microstructure configurations, which are achieved using three different synthesis methods, on the thermoelectric properties. Figure 7 shows the temperature dependence of the transport parameters of the $\mathrm{CoSb}_{3-x} \mathrm{Te}_{x}$ compounds prepared by the three methods. The temperature dependence of the electrical conductivity is depicted in Figure 7a. The electrical conductivity of all undoped samples of $\mathrm{CoSb}_{3}$ initially increases with temperature, reaches its maximum value near $600 \mathrm{~K}$ and is saturated. In contrast, all Te-doped structures display a decreasing electrical conductivity with increasing temperature, which 
a
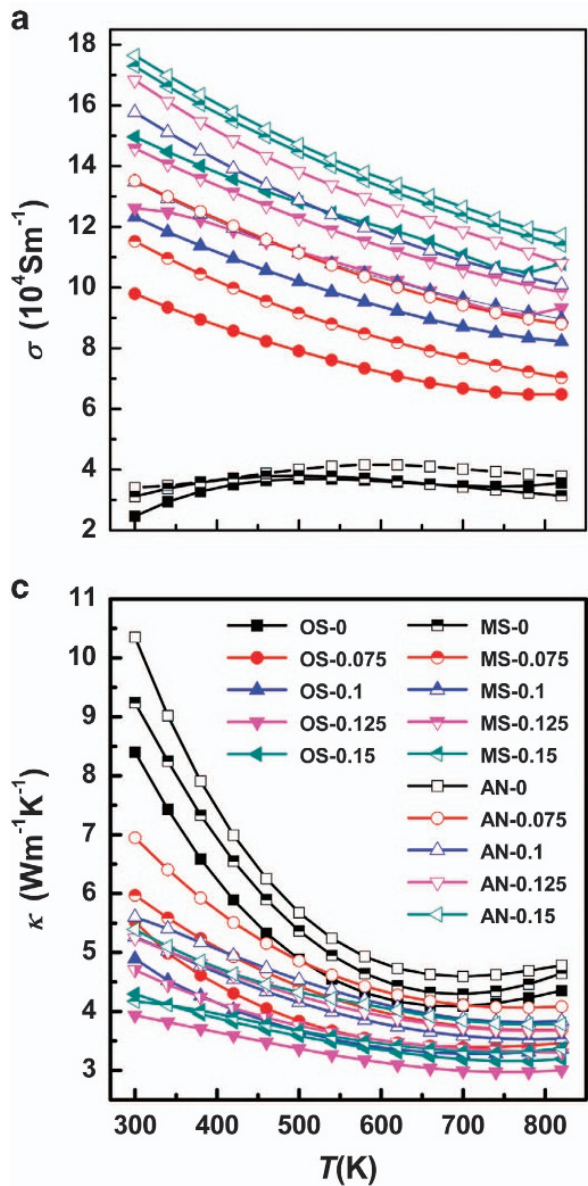

b

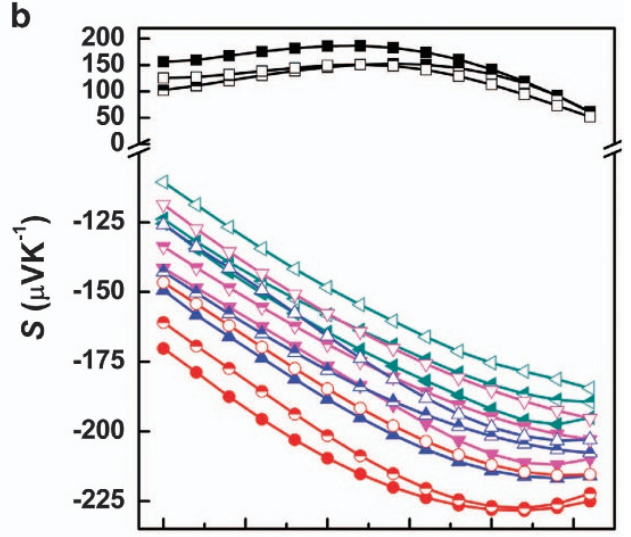

d

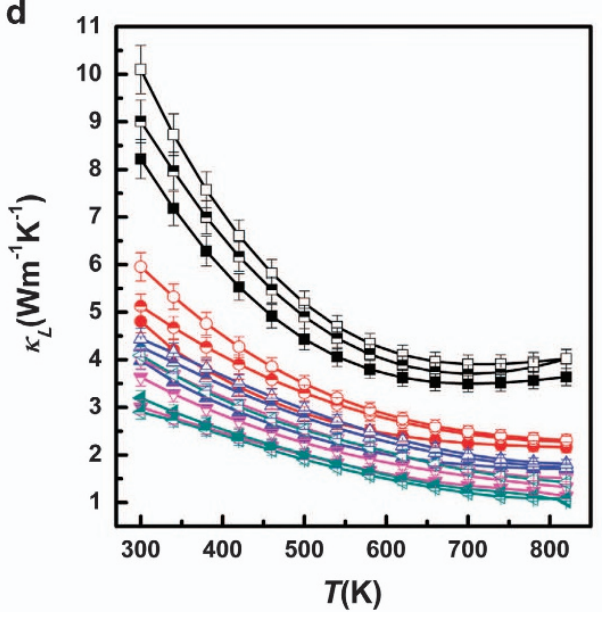

Figure 7 Temperature dependence of transport properties of $\mathrm{CoSb}_{3-x} \mathrm{Te} \mathrm{e}_{x}$ compounds prepared by the three different methods. (a) Electrical conductivity; (b) Seebeck coefficient; (c) total thermal conductivity; (d) lattice thermal conductivity.

is a characteristic of degenerate semiconductors. As we know, the Te atom has one more outer electron than $\mathrm{Sb}$ and can donate one extra electron to the conduction band when Te substitutes for Sb. Hence, the electrical conductivity of the samples prepared by the identical method significantly increases with increasing Te content, as expected from a progressively increased carrier concentration.

Figure $7 \mathrm{~b}$ illustrates the temperature dependence of the Seebeck coefficient $S$. In the entire temperature range covered, the Seebeck coefficient of all undoped $\mathrm{CoSb}_{3}$ samples is positive, which indicates the dominance of holes in their transport behavior. Te doping has a dramatic effect on the Seebeck coefficient and converts the structures into n-type conductors because the Te that substitutes for $\mathrm{Sb}$ acts as a donor. As expected, with the decreasing Te content, the magnitude of the Seebeck coefficient of $\mathrm{CoSb}_{3-x} \mathrm{Te}_{x}$ samples prepared by this method significantly increases. At the identical nominal doping level, the samples prepared by the OS-PAS method have higher absolute values of $S$ than the samples prepared by the other two methods. For example, when $x=0.125$, the absolute value of the Seebeck coefficient of the OS-PAS-prepared sample is $210 \mu \mathrm{V} \mathrm{K}^{-1}$ at $820 \mathrm{~K}$, whereas the values of the samples prepared with the MS-PAS and MQ-AN-PAS techniques are 202 and $194 \mu \mathrm{V} \mathrm{K}^{-1}$, respectively. The OS-PASprepared sample has a marginally higher magnitude of the Seebeck coefficient because of its lower carrier density, which is consistent with its lowest electrical conductivity. Supplementary Figure S5 shows the Seebeck coefficient and carrier mobility at room temperature as functions of the carrier concentration of the synthesized $\mathrm{CoSb}_{3-x} \mathrm{Te}_{x}$ compounds using the three methods. The temperature dependence of the power factor is shown in Supplementary Figure S6.

The thermal conductivity of all samples gradually decreases with increasing temperature, as shown in Figure $7 \mathrm{c}$. In the undoped samples above $\sim 650 \mathrm{~K}$, the thermal conductivity begins to rise, which signals the onset of intrinsic excitations (bipolar effect). Upon Te doping, the thermal conductivity decreases, but the samples prepared by the same method maintain similar trends. The samples prepared by OS-PAS have lower thermal conductivity than the same samples prepared by the other two synthesis techniques. For example, the room temperature thermal conductivity of pure $\mathrm{CoSb}_{3}$ prepared by OS-PAS is $8.4 \mathrm{~W} \mathrm{~m}^{-1} \mathrm{~K}^{-1}$, which represents a decrease of approximately $10 \%$ and $23 \%$ compared with pure $\mathrm{CoSb}_{3}$ prepared by MS-PAS and MQ-AN-PAS, respectively.

To eliminate the effect of the electronic thermal conductivity, we also show the behavior of the lattice thermal conductivity $\kappa_{\mathrm{L}}$ in Figure $7 \mathrm{~d}$. The lattice thermal conductivity was obtained from the total thermal conductivity by subtracting the electronic part using the Wiedemann-Franz law with the Lorenz number of $2.45 \times 10^{-8} \mathrm{~W} \mathrm{~K}^{-2}$. The lattice thermal conductivities of all samples decrease with the increasing temperature, which reflects the dominant role of phonon umklapp processes. The presence of Te in the structure introduces intense point defect scattering, and the thermal conductivity decreases. The room temperature value of $\kappa_{\mathrm{L}}$ of $\mathrm{CoSb}_{2.875} \mathrm{Te}_{0.125}$ 

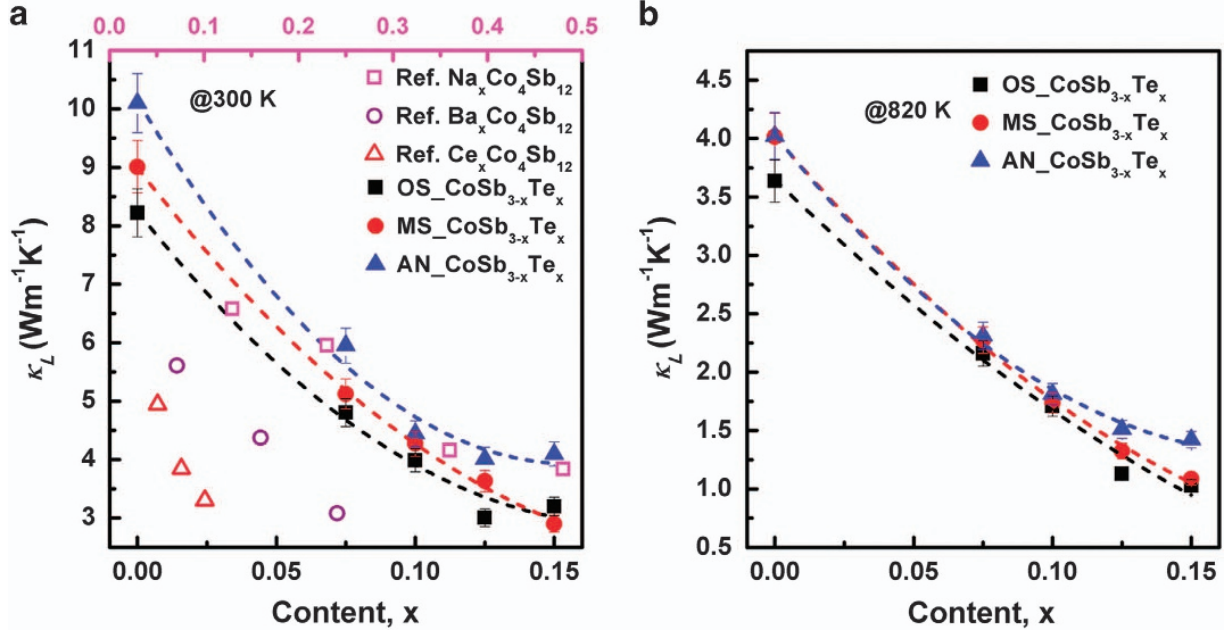

Figure 8 Lattice thermal conductivity at 300 and $820 \mathrm{~K}$ as a function of the content of Te for samples fabricated by the three different methods. (a) Lattice thermal conductivities at $300 \mathrm{~K}$ including for comparison the data for skutterudites single-filled with alkali, alkaline-earth and rare earth metals. For filled skutterudites, use the scale at the top of $\mathbf{b}$ Lattice thermal conductivity measured at $820 \mathrm{~K}$ for $\mathrm{CoSb}_{3-x} \mathrm{Te}_{x}$ samples prepared by the three different techniques. The dashed lines are to guide the eye.

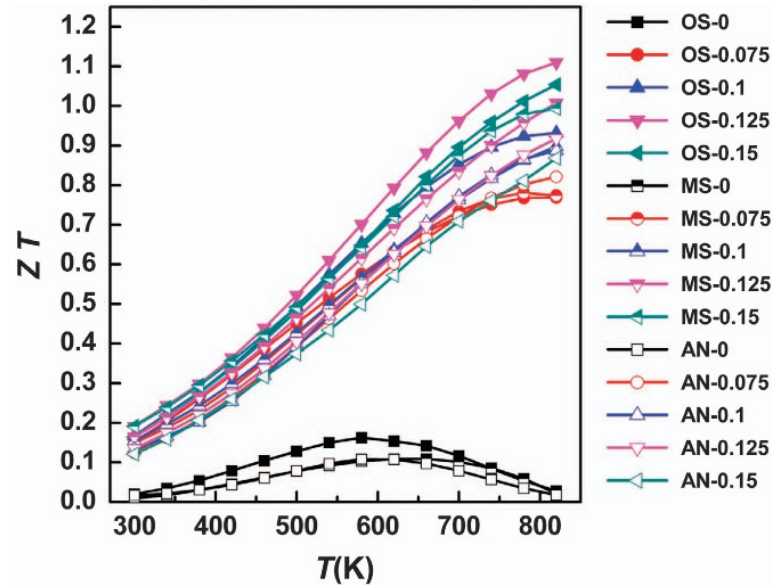

Figure 9 Temperature dependence of $Z T$ for $\operatorname{CoSb}_{3-x} T e_{x}$ compounds prepared by the three different methods.

prepared by OS-PAS is $3.0 \mathrm{~W} \mathrm{~m}^{-1} \mathrm{~K}^{-1}$, which decreases by $>60 \%$ compared with pure $\mathrm{CoSb}_{3}$. Again, the sample prepared with the OS-PAS process has a lower lattice thermal conductivity than those prepared by the other two techniques, which demonstrates that their hierarchical structure (that is, atomic scale, nanoscale and mesoscale) more effectively scatters phonons.

Figure 8 displays the lattice thermal conductivity $\kappa_{\mathrm{L}}$ at 300 and $820 \mathrm{~K}$ as a function of the Te content for samples fabricated by different methods. As shown in Figure 8a, the samples synthesized by the OS-PAS technique have smaller $\kappa_{\mathrm{L}}$ than the (nanostructured) samples prepared by MS-PAS and samples with the $\mu \mathrm{m}$-scale grains fabricated by the MQ-AN-PAS technique. Perhaps structures with multi-nanometer-scale imperfections scatter acoustic phonons over a wider frequency range and more significantly decrease $\kappa_{\mathrm{L}}$ than the structures with a notably narrow nanoscale distribution of imperfections. To highlight the higher effectiveness of the OS-PAS technique in decreasing $\kappa_{\mathrm{L}}$, we compare in Figure 8a our room temperature value of the lattice thermal conductivity with the reported values for alkali-filled, alkaline earth-filled and rare earth-filled skutterudites. The coordinate axis of the data, which are represented by hollow symbols (all single-filled skutterudites), is at the top of the figure. $\mathrm{CoSb}_{2.875} \mathrm{Te}{ }_{0.125}$ synthesized by the OS-PAS technique has much lower $\kappa_{\mathrm{L}}$ than $\mathrm{Na}_{0.48} \mathrm{Co}_{4} \mathrm{Sb}_{12}{ }^{35}$ and a notably close $\kappa_{\mathrm{L}}$ value to $\mathrm{Ba}_{0.23} \mathrm{Co}_{4} \mathrm{Sb}_{12},{ }^{36}$ which indicates that the lattice thermal conductivity obtained using this method can be as low as that achieved with most single-filled skutterudites. $\kappa_{\mathrm{L}}$ at $820 \mathrm{~K}$ as a function of the Te content is presented in Figure $8 \mathrm{~b}$. Again, the lattice thermal conductivity $\kappa_{\mathrm{L}}$ achieved by the OS-PAS technique is lower than $\kappa_{\mathrm{L}}$ of samples fabricated by the other two techniques. When the Te content is $x=0.125$, the lattice thermal conductivity of the OS-PAS sample is only $1.13 \mathrm{~W} \mathrm{~m}^{-1} \mathrm{~K}^{-1}$ at $820 \mathrm{~K}$; this notably low value will benefit the high-temperature thermoelectric performance and improve the figure of merit $Z T$.

Figure 9 shows the temperature dependence of $Z T$ for $\mathrm{CoSb}_{3-x} \mathrm{Te}_{x}$ compounds prepared with the three methods. Undoped $\mathrm{CoSb}_{3}$ has a notably low $Z T$, which peaks near $600 \mathrm{~K}$ with $Z T \sim 0.1-0.16$; the sample prepared with the OS-PAS method had the highest value. With the Te doping, the figure of merit dramatically increases to $0.7-1.1$ at approximately $800 \mathrm{~K}$ depending on the Te content and synthesis method. The highest $Z T$ value is obtained with $\mathrm{CoSb}_{2.875} \mathrm{Te}_{0.125}$ fabricated by OS-PAS (approximately 1.1 at $820 \mathrm{~K}$ ). This value is an increase of $10 \%$ and $21 \%$ compared with the samples prepared with the MS-PAS and MQ-AN-PAS techniques, respectively. Such ZT values are much higher than the figure of merit of samples prepared by the traditional method ${ }^{37}$ and mechanical alloying ${ }^{20}$ method. The higher ZT values of all OS-PAS-prepared samples reflect their hierarchical structures, which most effectively scatter a broad range of phonon frequencies and reduce the lattice thermal conductivity.

\section{DISCUSSION}

Considering that $\mathrm{CoSb}_{3}$ synthesized by OS-PAS at a sufficiently high sintering temperature $\left(\sim 550^{\circ} \mathrm{C}\right)$ is a single phase and has a relatively small grain structure, we focus on the key factors that affect the formation of the single phase and yield fine grains in $\mathrm{CoSb}_{3}$-based skutterudites. To eliminate the effect of the grain size distribution in the starting powders of $\mathrm{Co}$ and $\mathrm{Sb}$, all precursor powders were sieved through 200 mesh. Figure 10 shows the morphology of $\mathrm{CoSb}_{3}$ samples that were prepared by different synthesis techniques and under 
different PAS processing conditions. Figure 10a shows the FESEM image of $\mathrm{CoSb}_{3}$ synthesized using the MQ-AN-PAS technique with the sintering pressure of $40 \mathrm{MPa}$ at the sintering temperature of $650{ }^{\circ} \mathrm{C}$ and the holding time of $5 \mathrm{~min}$. Because this $\mathrm{CoSb}_{3}$ sample is a singlephase material after annealing, the sintering step merely densifies the structure and promotes the grain growth with no reactions or phase transformations. The grain structure of the MQ-AN-PAS sample is shown in Figure 10a and consists of grains of $2-15 \mu \mathrm{m}$. Figure $10 \mathrm{~b}$
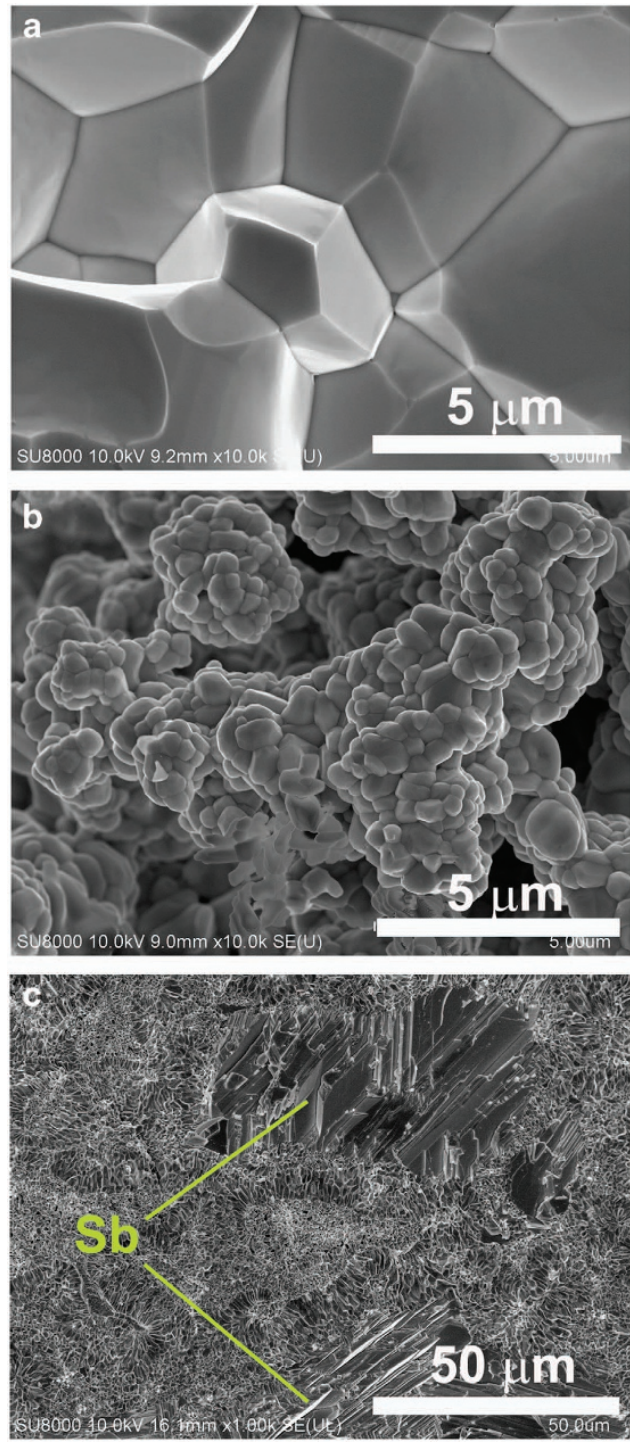

Figure 10 Morphology of $\mathrm{CoSb}_{3}$ samples prepared under different synthesis conditions. (a) FESEM image of $\mathrm{CoSb}_{3}$ synthesized by MQ-AN-PAS; (b, c) morphology of $\mathrm{CoSb}_{3}$ synthesized by OS-PAS with different sintering current and pressure: (b) current applied but no pressure used; (c) Sintering pressure of $40 \mathrm{MPa}$ but no current applied. presents the morphology of $\mathrm{CoSb}_{3}$ synthesized by OS-PAS when the sample was exposed for $5 \mathrm{~min}$ to a sintering temperature of $550{ }^{\circ} \mathrm{C}$, but no pressure $(0 \mathrm{MPa})$ was applied. The relevant microstructure contains pores, which indicates that the density of the material is low and that grains with a more spherical shape and sizes down to $200 \mathrm{~nm}$ tend to agglomerate into larger structures. Figure $10 \mathrm{c}$ shows the morphology of $\mathrm{CoSb}_{3}$ synthesized using the OS-PAS method under the pressure of $40 \mathrm{MPa}$ at the sintering temperature of $550{ }^{\circ} \mathrm{C}$ and the holding time of $5 \mathrm{~min}$, but no current passed through the ingot when the graphite die and starting powder were separated with insulating thick ceramic plates. The XRD patterns in Supplementary Figure S7 and the EDX results (regions of Sb identified in Figure 10c) show that the product is not single-phase $\mathrm{CoSb}_{3}$ because some fraction of $\mathrm{Sb}$ remained unreacted and trace amounts of CoSb were also detected. This result clearly demonstrates that the formation of the $\mathrm{CoSb}_{3}$ phase significantly benefits from the passage of the electric current in the sintering process.

The morphology characteristics in Figures $10 \mathrm{a}-\mathrm{c}$ are listed in Table 1. The results show that the grain size is coarse ( up to $15 \mu \mathrm{m}$ ) when the sintered powder is the $\mathrm{CoSb}_{3}$ phase. When the PAS process is applied to a powder of $\mathrm{Co}$ and $\mathrm{Sb}$ (stoichiometric quantities), the resulting grain structure is notably small (approaching $200 \mathrm{~nm}$ ). The use of a current during the sintering process promotes the formation of a single-phase $\mathrm{CoSb}_{3}$ structure. The effect of pressure is to achieve samples with high density. It has been reported that the current assists to accelerate the atomic diffusion; this effect is referred to as the 'electronic wind'. ${ }^{38}$ The current may also decrease the nucleation barrier of a newly forming phase and enhance the rate of nucleation. ${ }^{27-29}$

In summary, we demonstrated a rapid OS-PAS processing technique to fabricate $\mathrm{CoSb}_{3}$-based skutterudite materials. The technique facilitates the formation of a pure $\mathrm{CoSb}_{3}$ phase directly from the starting elemental powders of $\mathrm{Co}$ and $\mathrm{Sb}$ if the sintering temperature is at least $550{ }^{\circ} \mathrm{C}$. The PAS processing, that is, the passage of the current during the sintering process, assists in the formation of a pure skutterudite phase. The entire OS-PAS synthesis takes less than an hour and bypasses undesired thermodynamically favored phases, such as $\mathrm{CoSb}_{2}$. The OS-PAS synthesis of $\mathrm{CoSb}_{3}$-based skutterudites produces a hierarchical structure with scales from atomic size to nearly $15 \mu \mathrm{m}$. Such multilength-scale structures scatter phonons over a broad range of frequencies and significantly decrease the thermal conductivity compared with samples fabricated using other techniques. The highest $Z T$ of 1.1 at $820 \mathrm{~K}$ is obtained with a Te-doped skutterudite of composition $\mathrm{CoSb}_{2.875} \mathrm{Te}_{0.125}$, which was prepared using the OS-PAS technique. This high $Z T$ value with only a doped skutterudite rivals the performance of single-filled skutterudites, whose preparation is more expensive and far more time consuming. This newly developed OS-PAS technique provides a synthesis route, with which one can rapidly fabricate multiscale thermoelectric materials with excellent TE properties at a notably low cost. Thus the technique is eminently suited for mass production and opens the road to commercial applications of skutterudites. Attempts should be made to

Table 1 Morphology characteristics corresponding to FESEM images in Figures 10a-c

\begin{tabular}{|c|c|c|c|c|c|}
\hline Different process & Compositions being sintered & Sintering current & Sintering pressure & Single phase & Grain size \\
\hline MQ-AN-PAS & $\mathrm{CoSb}_{3}$ & Yes & Yes & Yes & $>5 \mu \mathrm{m}$ \\
\hline OS-PAS & $\mathrm{Co}+3 \mathrm{Sb}$ & Yes & No & Yes & $200 \mathrm{~nm} \sim 3 \mu \mathrm{m}$ \\
\hline OS-PAS & $\mathrm{Co}+3 \mathrm{Sb}$ & No & Yes & No & $200 \mathrm{~nm} \sim 1 \mu \mathrm{m}$ \\
\hline
\end{tabular}

Abbreviations: FESEM, field-emission scanning electron microscope; OS-PAS, one-step plasma-activated sintering. 
apply the technique to fabricate filled skutterudite structures, which may have even better thermoelectric properties.

\section{CONFLICT OF INTEREST}

The authors declare no conflict of interest.

\section{ACKNOWLEDGEMENTS}

This work is financially supported by the National Basic Research Program of China under project 2013CB632502, the Natural Science Foundation of China (Grant Nos 51172174, 51402222, 51521001, 51632006), the 111 Project of China (Grant No B07040) and the CERC-CVC joint US-China Program supported by the US Department of Energy under Award Number DE-PI0000012. We thank Tingting Luo and Rong Jiang for their HRTEM analysis in the Materials Research and Test Center of WUT.

1 Biswas, K., He, J., Blum, I. D., Wu, C.-I., Hogan, T. P., Seidman, D. N., Dravid, V. P. \& Kanatzidis, M. G. High-performance bulk thermoelectrics with all-scale hierarchical architectures. Nature 489, 414-418 (2012).

2 Pei, Y., Shi, X., LaLonde, A., Wang, H., Chen, L. \& Snyder, G. J. Convergence of electronic bands for high performance bulk thermoelectrics. Nature 473, 66-69 (2011).

3 Su, X., Fu, F., Yan, Y., Zheng, G., Liang, T., Zhang, Q., Cheng, X., Yang, D., Chi, H. \& Tang, $X$. Self-propagating high-temperature synthesis for compound thermoelectrics and new criterion for combustion processing. Nat. Commum. 5, 4908-4914 (2014).

4 Snyder, G. J., Christensen, M., Nishibori, E., Caillat, T. \& Iversen, B. B. Disordered zinc in Zn4Sb3 with phonon-glass and electron-crystal thermoelectric properties. Nat. Mater. 3, 458-463 (2004).

5 Zhao, L.-D., Lo, S.-H., Zhang, Y., Sun, H., Tan, G., Uher, C., Wolverton, C., Dravid, V. P. \& Kanatzidis, M. G. Ultralow thermal conductivity and high thermoelectric figure of merit in SnSe crystals. Nature 508, 373-377 (2014).

6 Sales, B., Mandrus, D. \& Williams, R.K. Filled skutterudite antimonides: a new class of thermoelectric materials. Science 272, 1325-1328 (1996).

7 Su, X., Li, H., Wang, G., Chi, H., Zhou, X., Tang, X., Zhang, Q. \& Uher, C. Structure and transport properties of double-doped $\mathrm{CoSb}_{2.75} \mathrm{Ge}_{0.25-\mathrm{x}} \mathrm{Te}_{\mathrm{x}}(\mathrm{x}=0.125-0.20)$ with in situ nanostructure. Chem. Mater. 23, 2948-2955 (2011).

8 Rogl, G. \& Rogl, P. Mechanical properties of skutterudites. Sci. Adv. Mater. 3, 517-538 (2011).

9 Yang, J. \& Stabler, F. R. Automotive applications of thermoelectric materials. J. Electron. Mater. 38, 1245-1251 (2009).

10 Salzgeber, K., Prenninger, P., Grytsiv, A., Rogl, P. \& Bauer, E. Skutterudites: thermoelectric materials for automotive applications? J. Electron. Mater. 39, 2074-2078 (2010).

11 Liang, T., Su, X., Yan, Y., Zheng, G., Zhang, Q., Chi, H., Tang, X. \& Uher, C. Ultra-fast synthesis and thermoelectric properties of Te doped skutterudites. J. Mater. Chem. A 2, 17914-17918 (2014)

12 Shi, X., Yang, J., Salvador, J. R., Chi, M., Cho, J. Y., Wang, H., Bai, S., Yang, J., Zhang, W. \& Chen, L. Multiple-filled skutterudites: high thermoelectric figure of merit through separately optimizing electrical and thermal transports. J. Am. Chem. Soc. 133, 7837-7846 (2011)

13 Callaway, J. Model for lattice thermal conductivity at low temperatures. Phys. Rev. 113, 1046 (1959).

14 Sales, B., Chakoumakos, B., Mandrus, D. \& Sharp, J. Atomic displacement parameters and the lattice thermal conductivity of clathrate-like thermoelectric compounds. J. Solid State Chem. 146, 528-532 (1999).

15 Roufosse, M. C. \& Klemens, P. Lattice thermal conductivity of minerals at high temperatures. J. Geophys. Res. 79, 703-705 (1974).

16 Guo, R., Wang, X. \& Huang, B. Thermal conductivity of skutterudite $\mathrm{CoSb}_{3}$ from first principles: Substitution and nanoengineering effects. Sci. Rep. 5, 7806 (2015).

17 Zebarjadi, M., Yang, J., Lukas, K., Kozinsky, B., Yu, B., Dresselhaus, M. S., Opeil, C., Ren, Z. \& Chen, G. Role of phonon dispersion in studying phonon mean free paths in skutterudites. J. Appl. Phys. 112, 044305 (2012)

18 Kitagawa, H., Wakatsuki, M., Nagaoka, H., Noguchi, H., Isoda, Y., Hasezaki, K. \& Noda, Y. Temperature dependence of thermoelectric properties of $\mathrm{Ni}$-doped $\mathrm{CoSb} 3$. J. Phys. Chem. Solids 66, 1635-1639 (2005).
19 Peng, J., Yang, J., Song, X., Chen, Y. \& Zhang, T. Effect of Fe substitution on the thermoelectric transport properties of CoSb3-based skutterudite compound. J. Alloys Compd. 426, 7-11 (2006).

20 Liu, W.-S., Zhang, B.-P., Li, J.-F., Zhang, H.-L. \& Zhao, L.-D. Enhanced thermoelectric properties in CoSb3-xTex alloys prepared by mechanical alloying and spark plasma sintering. J. Appl. Phys. 102, 103717 (2007).

21 Su, X., Li, H., Yan, Y., Wang, G., Chi, H., Zhou, X., Tang, X., Zhang, Q. \& Uher, C. Microstructure and thermoelectric properties of $\mathrm{CoSb}_{2.75} \mathrm{Ge}_{0.25-\mathrm{x}} \mathrm{Tex}$ prepared by rapid solidification. Acta Mater. 60, 3536-3544 (2012).

22 Shi, X., Zhang, W., Chen, L. \& Yang, J. Filling fraction limit for intrinsic voids in crystals: doping in skutterudites. Phys. Rev. Lett. 95, 185503 (2005).

$23 \mathrm{Li}, \mathrm{H}$., Tang, X., Zhang, Q. \& Uher, C. Rapid preparation method of bulk nanostructured $\mathrm{Yb}_{0.3} \mathrm{CO}_{4} \mathrm{Sb}_{12+y}$ compounds and their improved thermoelectric performance. Appl. Phys. Lett. 93, 252109 (2008).

$24 \mathrm{Li}, \mathrm{H}$., Tang, X., Zhang, Q. \& Uher, C. High performance $\mathrm{In}_{\mathrm{x}} \mathrm{Ce}_{\mathrm{y}} \mathrm{Co}_{4} \mathrm{Sb}_{12}$ thermoelectric materials with in situ forming nanostructured InSb phase. Appl. Phys. Lett. 94, 102114 (2009).

25 Zhao, L.-D., Dravid, V. P. \& Kanatzidis, M. G. The panoscopic approach to high performance thermoelectrics. Energy Environ. Sci. 7, 251-268 (2014).

26 Zhao, L., Wu, H., Hao, S., Wu, C.-I., Zhou, X., Biswas, K., He, J., Hogan, T. P., Uher, C. \& Wolverton, C. All-scale hierarchical thermoelectrics: MgTe in PbTe facilitates valence band convergence and suppresses bipolar thermal transport for high performance. Energy Environ. Sci. 6, 3346-3355 (2013).

27 Zhou, Y., Guo, J., Zhang, W. \& He, G. Influence of electropulsing on nucleation during phase transformation. J. Mater. Res. 17, 3012-3014 (2002).

28 Dolinsky, Y. \& Elperin, T. Thermodynamics of phase transitions in current-carrying conductors. Phys. Rev. B 47, 14778 (1993).

29 Dolinsky, Y. \& Elperin, T. Phase transitions in fractal clusters in the presence of electric fields. Phys. Rev. B 62, 12656 (2000).

30 Guillon, O., Gonzalez-Julian, J., Dargatz, B., Kessel, T., Schierning, G., Räthel, J. \& Herrmann, M. Field-assisted sintering technology/spark plasma sintering: mechanisms, materials, and technology developments. Adv. Eng. Mater. 16, 830-849 (2014).

31 Ishida, K. \& Nishizawa, T. The Co-Sb (Cobalt-Antimony) system. Bull. Alloy Phase Diag. 11, 243-248 (1990)

32 Liang, T., Su, X., Tan, X., Zheng, G., She, X., Yan, Y., Tang, X. \& Uher, C. Ultra-fast nonequilibrium synthesis and phase segregation in $\ln _{x} S_{1-x} T_{e}$ thermoelectric by SHS-PAS processing. J. Mater. Chem. C 3, 8550-8558 (2015).

33 Zheng, Y., Zhang, Q., Su, X., Xie, H., Shu, S., Chen, T., Tan, G., Yan, Y., Tang, X. \& Uher, C. Mechanically robust BiSbTe alloys with superior thermoelectric performance: a case study of stable hierarchical nanostructured thermoelectric materials. Adv. Energy Mater. 5, 1401391-1401401 (2015).

34 Zou, T., Qin, X., Li, D., Sun, G., Dou, Y., Wang, Q., Ren, B., Zhang, J., Xin, H. \& Li, Y. Simultaneous enhancement in thermoelectric power factor and phonon blocking in hierarchical nanostructured $\beta-\mathrm{Zn}_{4} \mathrm{Sb}_{3}-\mathrm{Cu}_{3} \mathrm{SbSe}$. Appl. Phys. Lett. 104, 013904 (2014)

35 Pei, Y., Yang, J., Chen, L., Zhang, W., Salvador, J. \& Yang, J. Improving thermoelectric performance of caged compounds through light-element filling. Appl. Phys. Lett. 95, 042101 (2009).

36 Chen, L., Kawahara, T., Tang, X., Goto, T., Hirai, T., Dyck, J., Chen, W. \& Uher, C. Anomalous barium filling fraction and n-type thermoelectric performance of $\mathrm{Ba}_{\mathrm{y}} \mathrm{Co}_{4} \mathrm{Sb}_{12}$. J. Appl. Phys. 90, 1864-1868 (2001).

$37 \mathrm{Li}$, X., Chen, L., Fan, J., Zhang, W., Kawahara, T. \& Hirai, T. Thermoelectric properties of Te-doped CoSb3 by spark plasma sintering. J. Appl. Phys. 98, 083702 (2005).

38 Sprecher, A., Mannan, S. \& Conrad, H. Overview no. 49: On the mechanisms for the electroplastic effect in metals. Acta Metall. 34, 1145-1162 (1986).

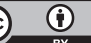

This work is licensed under a Creative Commons Attribution 4.0 International License. The images or other third party material in this article are included in the article's Creative Commons license, unless indicated otherwise in the credit line; if the material is not included under the Creative Commons license, users will need to obtain permission from the license holder to reproduce the material. To view a copy of this license, visit http:// creativecommons.org/licenses/by/4.0/

(C) The Author(s) 2017

Supplementary Information accompanies the paper on the NPG Asia Materials website (http://www.nature.com/am) 